\title{
Simulation of Clipping Position for Cerebral Aneurysms Using Three-dimensional Computed Tomography Angiography
}

\author{
Kazuya FUTAMI, Mitsutoshi NAKADA, Masayuki IWATO, Daisuke KITA, \\ Tadao MIYAMORI, and Junkoh YAMASHITA*
}

\begin{abstract}
Department of Neurosurgery, Toyama City Hospital, Toyama; *Department of Neurosurgery, Division of Neuroscience, Graduate School of Medical Science, Kanazawa University, Kanazawa, Ishikawa
\end{abstract}

\begin{abstract}
A novel method for the simulation of the clipping position for cerebral aneurysms based on threedimensional computed tomography (3D CT) angiography was evaluated. Rotating the regional 3D CT angiography images including the aneurysm provided the virtual intraoperative views of 36 cerebral aneurysms that were eligible for clipping through a pterional approach with a perpendicularly applied straight clip. The cut-along-trace function of the 3D CT workstation was used to simulate the clipping position. The presence or absence of aneurysm remnants was preoperatively evaluated by observing the clipping simulation image. Intraoperative endoscopy and postoperative cerebral angiography were routinely performed to confirm the completeness of obliterations. Nineteen of 21 aneurysms for which complete obliteration was preoperatively expected were confirmed to have no aneurysm remnant. Nine of 15 aneurysms which were expected to have aneurysm remnant were confirmed to persist. The clipping simulation images could correctly predict aneurysm remnant after the initial clipping with a sensitivity of $90.5 \%$ and specificity of $60 \%$. The present simulation method can predict aneurysm remnants and improve the likelihood of complete obliteration by clipping.
\end{abstract}

Key words: cerebral aneurysm, clipping, simulation, three-dimensional computed tomography angiography

\section{Introduction}

The incidence and the long-term outcome of aneurysm remnants after clipping are now of greatest concern, especially for comparing the results of clipping and endovascular coiling. ${ }^{24)}$ Postoperative angiography after clipping of cerebral aneurysms showed that the incidence of residual aneurysms was $2.3 \%$ to $14 \%{ }^{24)}$ Aneurysm remnants after clipping may regrow and lead to recurrent symptoms of hemorrhage or mass effect. $4,5,7-9,11,14$, 16-21,24) Therefore, aneurysm surgery should aim at complete circulatory exclusion of aneurysms, but this cannot be achieved in several circumstances including large necks, insufficient exposure, and mal-positioning of clips. . $^{4,7,9,20)}$

Aneurysm remnants can be classified into two groups, dog-ear and broad-based remnant. ${ }^{5)}$ Two of

Received February 12, 2003; Accepted August 26, 2003 eight dog-ear remnants and three of four broadbased remnants grew in the follow-up period ranged 2.6 to 9.7 years. ${ }^{5}$ ) The risk of hemorrhage from aneurysm remnant has been estimated at approximately $0.5 \%$ to $1.9 \%$ per year. ${ }^{5,8)}$ In addition, reoperation for aneurysm remnants presents technical difficulties ${ }^{9,16)}$ and high morbidity and mortality. ${ }^{7)}$

The anatomy behind an aneurysm and its parent arteries may be difficult to determine, so failure to select the optimal clip can lead to mal-positioning of clips. Simulation of clipping for cerebral aneurysms would certainly facilitate complete clipping. Threedimensional computed tomography (3D CT) angiography can provide virtual preoperative views to plan surgery for cerebral aneurysms. ${ }^{2,10,12,22,25,28)}$

The present study describes a cut-along-trace function using 3D CT angiography to simulate the clipping position for aneurysms with a perpendicularly applied straight clip, and prospectively evaluated the usefulness of this clipping simulation method. 


\section{Patients and Methods}

The present study included 34 patients, 12 men and 22 women, aged 35 to 84 years (mean 56.5 years) with 36 aneurysms including 21 ruptured aneurysms. All aneurysms had the fundus directed parallel to the plane of the virtual intraoperative view reconstructed using 3D CT angiography and the initial clipping was performed with a straight clip. Twenty-four aneurysms were located on the internal carotid artery (ICA)-posterior communicating artery (PCoA) bifurcation, seven on the anterior communicating artery, and five on the middle cerebral artery. All 36 aneurysms were $10 \mathrm{~mm}$ or less in size.

3D CT angiography was performed with a spiral CT scanner (ProSeed; GE-Yokogawa Medical Systems, Hino, Tokyo) with a section thickness of $1.0 \mathrm{~mm}$ and a table speed of $1.5 \mathrm{~mm} / \mathrm{sec}$. After determining the level of the circle of Willis by a preliminary thick slice scan, a dose of $80 \mathrm{ml}$ of non-ionic contrast material was injected intravenously with a power injector at the rate of $3.5-4.0 \mathrm{ml} / \mathrm{sec}$. Twenty seconds after the injection, CT scanning was commenced with parameters of $512 \times 512$ matrix, $120 \mathrm{kV}, 160 \mathrm{~mA}, 12.5 \mathrm{~cm}$ field of view, and reconstruction index of $1 \mathrm{~mm}$. Image reconstruction was performed on a General Electric Advantage Windows workstation.

The cerebral aneurysms were evaluated on the multi-directional 3D CT angiography images, then all structures except for bones and arteries adjacent to the aneurysms were removed by a manual cutaway technique. The virtual intraoperative view was reconstructed by rotating the image with a relatively small field of interest. A line was traced where the aneurysm could be definitely clipped with a straight clip. Using the cut-along-trace function of the 3D CT workstation, the image signals along the trace were deleted. The obtained image with the deleted zone was defined as the clipping simulation image.

The clipping simulation images were rotated to observe the blind corners between the neck and parent arteries to identify any potential aneurysm remnants after clipping. Aneurysm remnant was defined as a residual bulge on the clipping simulation image. The preoperative evaluations were performed by two of the authors (M.N. and M.I.), and a decision was made only if both reached the same conclusion.

All 36 aneurysms were clipped through a pterional approach, with the side based on the virtual intraoperative 3D CT angiography images. All 36 aneurysms were clipped by the same neurosurgeon (K.F.), who was unaware of the preopera- tive evaluations based on the clipping simulation images. All aneurysms were initially clipped with a straight clip, which was not intentionally applied as part of a staged application. ${ }^{27)}$ Immediately after the initial clipping, aneurysm remnants were evaluated by endoscopy. Any aneurysm remnant was treated by correction of the clip position or additional clipping to increase the completeness of obliteration. Routine postoperative conventional or digital subtraction cerebral angiography was performed.

The distribution of the $2 \times 2$ table of evaluations was analyzed by Fisher's exact test. $\mathrm{P}$ values less than 0.05 were considered significant.

\section{Results}

The preoperative evaluation of the clipping simulation images indicated complete obliteration of 21 aneurysms and remnants of 15 aneurysms. Endoscopic observations during surgery or postoperative cerebral angiography confirmed obliteration of 19 of the 21 aneurysms predicted to be completely excluded. Endoscopic observation during surgery showed complete obliteration in six aneurysms and aneurysm remnants in nine of the 15 aneurysms expected to persist. There was a strongly significant relationship between the aneurysm remnants predicted by the clipping simulation images and those confirmed using endoscopy (Fisher exact test, $\mathrm{p}=0.002$ ).

All aneurysms with remnants were located on ICA-PCoA bifurcations, and all remnants were located on the blind corner between the aneurysm and the parent arteries. The two remnant aneurysms among the 21 aneurysms expected to be completely obliterated had a wide aneurysm neck. Eleven aneurysm remnants were observed by endoscopy. Six of these 11 aneurysm remnants were completely obliterated by the additional application of a clip. The remaining five aneurysm remnants were wrapped with adhesive materials, because of compact adhesion with the surrounding perforating arteries in three cases and too small size to correct the clip position in the other two cases. Postoperative conventional cerebral angiography showed complete obliteration in all 36 aneurysms, except for two cases with compact adhesion of perforating arteries. The two cases of tiny aneurysm remnants were invisible.

\section{Case Illustrations}

Case 1: A 66-year-old female presented with subarachnoid hemorrhage. 3D CT angiography and conventional cerebral angiography revealed a right 

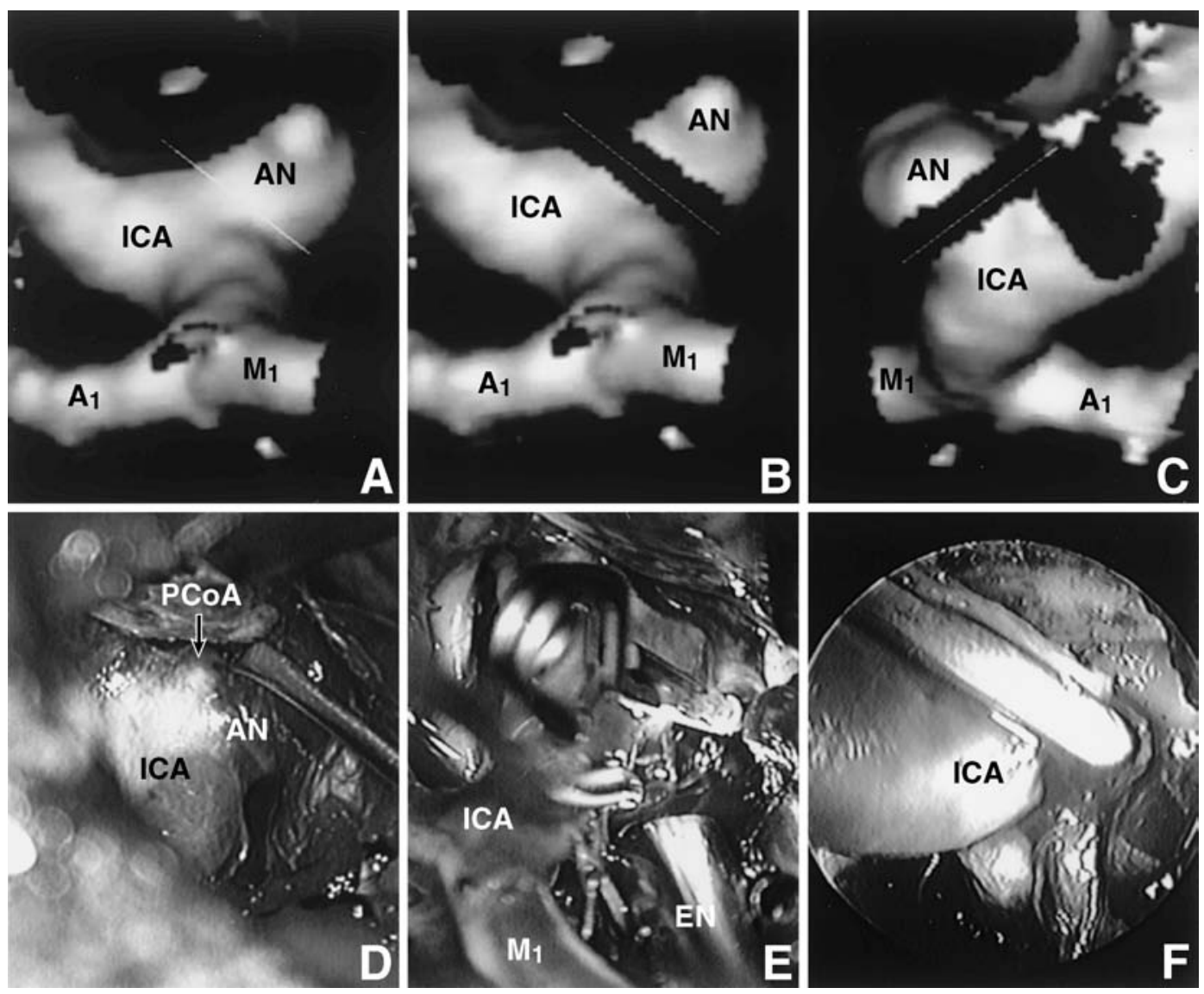

Fig. 1 Case 1 with a ruptured right internal carotid artery (ICA)-posterior communicating artery (PCoA) aneurysm (AN). A: Virtual intraoperative view based on three-dimensional computed tomography angiography showing the traced clipping position. B: Clipping simulation image reconstructed by deleting the image signals along the trace. C: Posterior-anterior view of the clipping simulation image indicating no aneurysm remnant after application of a straight clip. D, E: Intraoperative photographs before and after clipping, respectively. EN: endoscope. F: Endoscopic view showing complete obliteration.

ICA-PCoA aneurysm. The virtual intraoperative view was used to trace the line of the straight clip (Fig. 1A), and the clipping simulation image was reconstructed by deleting the image signals along the trace (Fig. 1B). Observation of the blind corner between the aneurysm and the ICA on the rotated image indicated no aneurysm remnant (Fig. 1C). Neck clipping was performed with a straight clip (Fig. 1D, E). Endoscopic observation showed the complete obliteration of the aneurysm (Fig. 1F) and preservation of the perforating arteries. Postoperative conventional cerebral angiography also showed complete obliteration. Her hospital course was uneventful.

Case 2: A 47-year-old female presented with right putaminal hemorrhage. Magnetic resonance angiography and conventional cerebral angiography incidentally revealed a left ICA-PCoA aneurysm. 3D
CT angiography showed the aneurysm had two domes projecting laterally and posteriorly, respectively (Fig. 2A, B). Observation of the blind corner by rotating the clipping simulation image indicated that the posteriorly projecting dome persisted (Fig. 2C). Endoscopic observation showed the dome remnant on the posterior wall behind the ICA after initial clipping with a straight clip (Fig. 2D, E). The dome was wrapped because of firm adhesion with the perforating arteries (Fig. 2F). Postoperative conventional cerebral angiography showed the dome remnant. The postoperative hospital course was uneventful.

Case 3: A 63-year-old female presented with subarachnoid hemorrhage. 3D CT angiography and conventional cerebral angiography revealed a left ICA-PCoA aneurysm. The virtual intraoperative view showed that the PCoA originated from the 

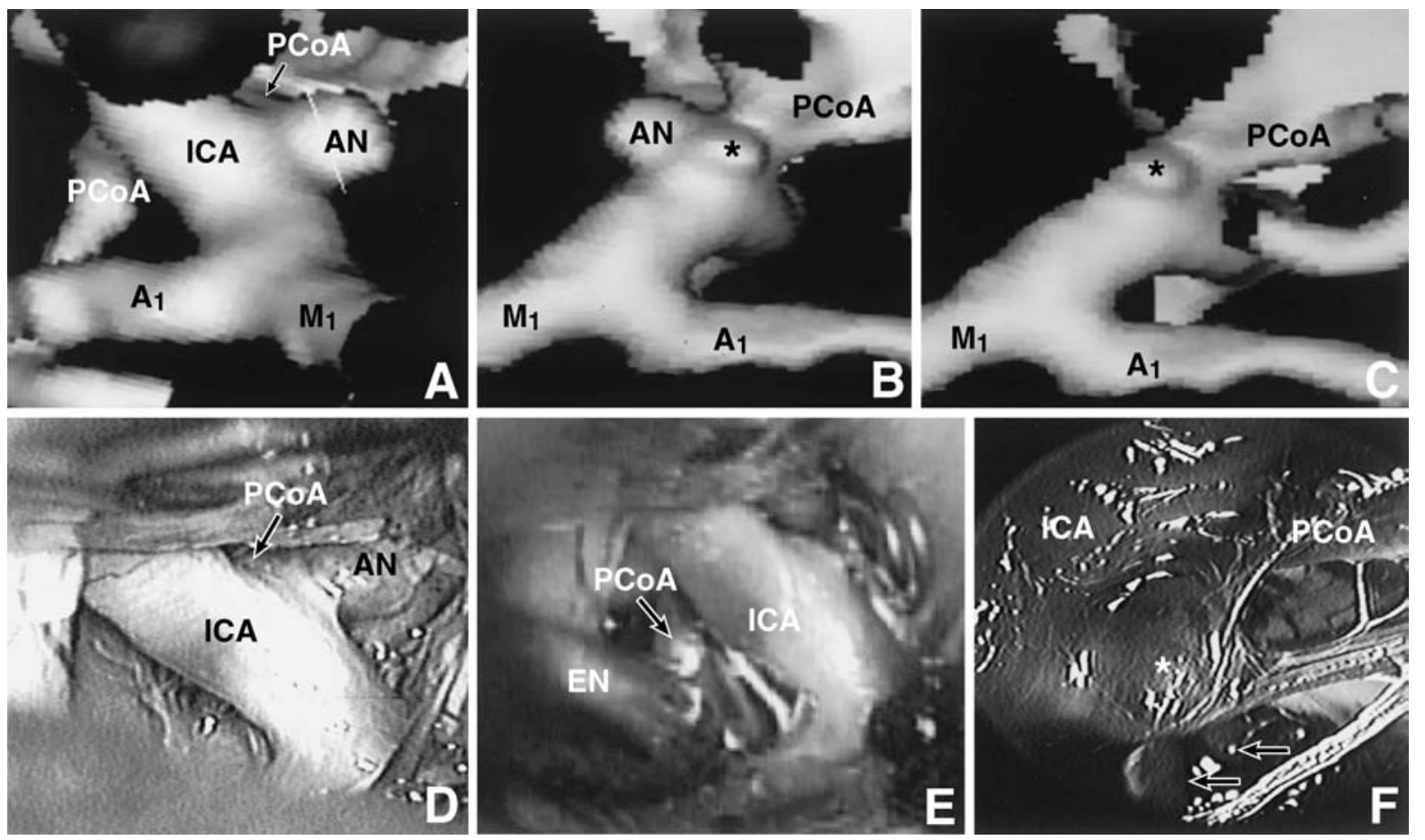

Fig. 2 Case 2 with an unruptured right internal carotid artery (ICA)-posterior communicating artery (PCoA) aneurysm (AN). A: Virtual intraoperative view based on three-dimensional computed tomography (3D CT) angiography showing the traced clipping position. B: Posterior-anterior view of the 3D CT angiography image showing an aneurysm with two domes projecting laterally and posteriorly (asterisk), respectively. C: Posterior-anterior view of the clipping simulation image indicating the posteriorly projecting dome (asterisk) remaining after application of a straight clip. D, E: Intraoperative photographs before and after clipping, respectively. EN: endoscope. F: Endoscopic view showing the posteriorly projecting dome (asterisk) remaining after clipping. Arrows indicate the tips of the clip.

aneurysm adjacent from the proximal neck. The predicted line of clipping was traced between the aneurysm just distal to the PCoA origin and the distal neck (Fig. 3A). Observation of the blind corner by rotating the clipping simulation image indicated a small aneurysm remnant (Fig. 3B). Intraoperative observation confirmed that the anterior choroidal artery as well as the PCoA originated from the aneurysm. Therefore, clipping was performed just distal to the origins of both arteries using a straight clip (Fig. 3C, D). Endoscopic observation after clipping showed a tiny aneurysm remnant remained as a "dog ear" on the blind corner (Fig. 3E), which was wrapped. Postoperative conventional cerebral angiography could not disclose the remnant.

\section{Discussion}

The present study showed that the cut-along-trace function of a 3D CT workstation can simulate the clipping position for cerebral aneurysms, if the aneurysm fundi are directed laterally to the parent artery in the operative fields and are eligible for clipping with a straight clip. Endoscopy during surgery and postoperative cerebral angiography confirmed the complete exclusion in 19 of 21 aneurysms for which complete obliteration was expected by the clipping simulation images. Endoscopic observations confirmed aneurysm remnants in nine of 15 aneurysms for which the clipping simulation images showed incomplete clipping. Therefore, the sensitivity and specificity of clipping simulation images to predict the incomplete clipping were $90.5 \%$ and $60 \%$, respectively.

Intraoperative angiography or endoscopy are presently used to evaluate the completeness of clipping during surgery. $1,3,6,13,15,17,23,26)$ However, intraoperative angiography has a complication rate of $1.7 \%$ to $3.7 \%$ and a false-negative rate of $1.8 \%$ to $5.2 \% .^{3,5,6,15)}$ In addition, intraoperative angiography does not give information about the status of any perforating arteries that may be inadvertently in- 

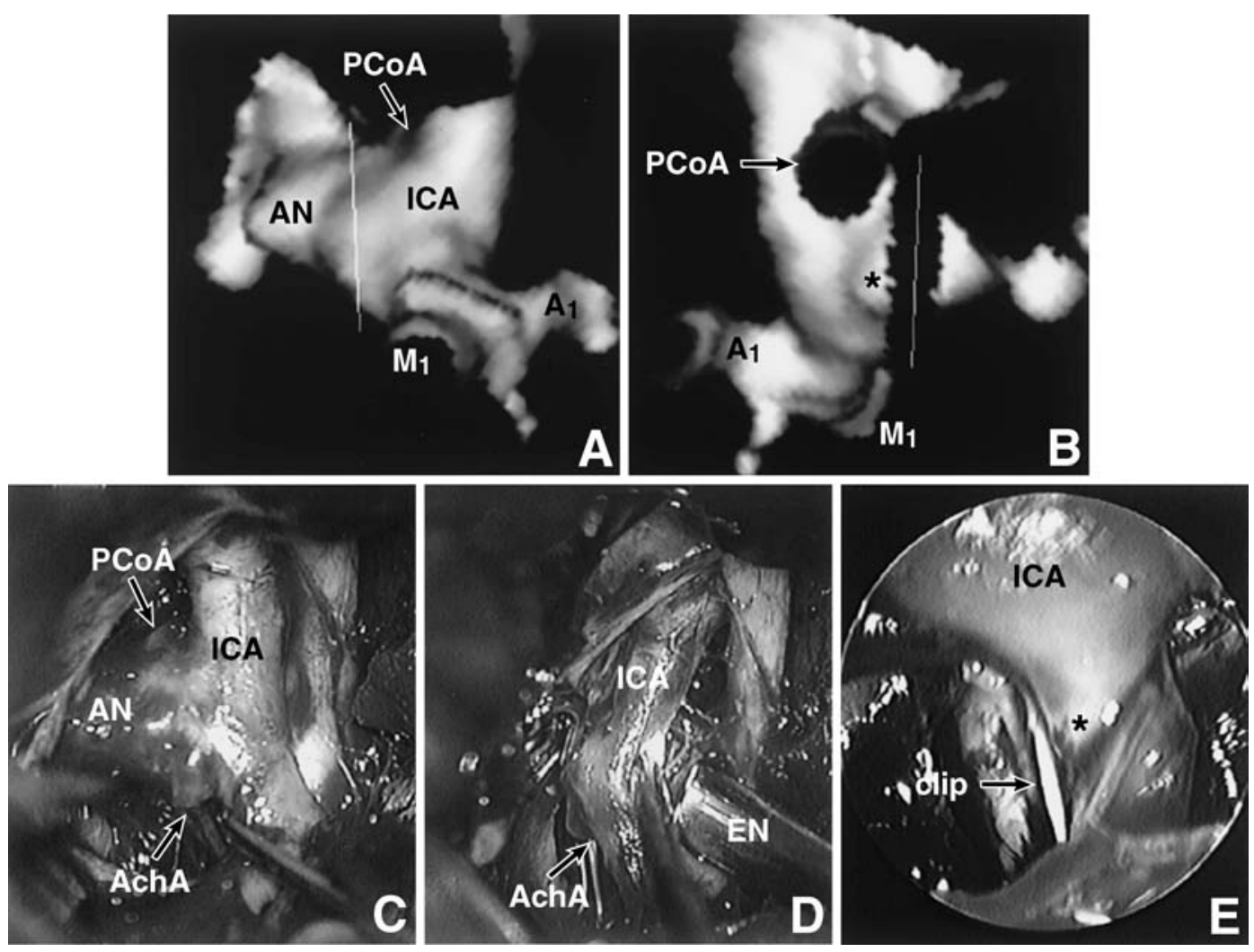

Fig. 3 Case 3 with a ruptured left internal carotid artery (ICA)-posterior communicating artery (PCoA) aneurysm (AN). A: Virtual intraoperative view based on three-dimensional computed tomography angiography showing the traced clipping position. B: Clipping simulation image rotated to observe the posterior surface of the ICA indicating a small aneurysm remnant (asterisk). C, D: Intraoperative photographs before and after clipping, respectively. AchA: anterior choroidal artery, EN: endoscope. E: Endoscopic view indicating a small aneurysm remnant as a "dog ear" (asterisk).

cluded in the clip blades. ${ }^{1)}$ The introduction of endoscopy into clipping surgery has some limitations. ${ }^{23)}$ Adequate space around the aneurysm is required to introduce the endoscope to avoid inadvertent trauma to surrounding structures. ${ }^{13)}$ Also, a clear operative field is prerequisite. ${ }^{23)}$ Therefore, endoscopy cannot be used in patients with severe subarachnoid hemorrhage. ${ }^{23)}$ The use of the endoscope may disturb microsurgical procedures. ${ }^{13)}$ The present method of prediction of incomplete clipping using clipping simulation images may be useful in deciding whether to perform intraoperative angiography or endoscopy.

In the present study, the incidence of incomplete clipping was $30.6 \%$ after initial clipping, $13.9 \%$ after the final procedure, and $5.6 \%$ by postoperative cerebral angiography. Except for the results of postoperative cerebral angiography, these were relatively high rates compared with previous incidences, ${ }^{5,8,18,24)}$ and may be attributed to various factors. First, in the present study, a small residual neck that may have been previously ignored was included as an aneurysm remnant. ${ }^{14)}$ Second, most aneurysms in the present study were located on the ICA-PCoA bifurcation, where the blind corner between the aneurysms and the ICA is difficult to confirm during surgery. ${ }^{1,4,19,20)}$ Additionally, the infundibulum of the PCoA partly implanted into the neck may prevent complete obliteration. ${ }^{20)}$ Many cases of regrowth and rerupture have been reported after clipping of ICA-PCoA aneurysms, ${ }^{4,11,16,19,21)}$ although the incidence of aneurysm remnants at this location differs. ${ }^{14,16,18,20)}$ Third, the present study included a thorough search for aneurysm remnants using endoscopy during surgery, especially around the blind corner between the aneurysm and its parent artery. Fourth, half of the aneurysms were unruptured. Especially for unruptured aneurysms, prevention of operative complications must take precedence over achieving complete obliteration, 
which might result in a relatively high incidence of incomplete clipping.

The present simulation method of the clipping position using the cut-along-trace function of a $3 \mathrm{D}$ CT workstation has several limitations. The selection of the clipping plane on the virtual intraoperative view must depend on the experience of the neurosurgeons or neuroradiologists. Distortion of the vascular structures by surgical manipulations cannot be modeled. 3D CT angiography cannot demonstrate small arteries including most perforating arteries because of the limited spatial resolution, ${ }^{2,10,25)}$ so preservation must be confirmed microsurgically. The virtual intraoperative view does not give adequate information about the structures around the aneurysm complex, such as the anterior clinoid process, tentorial edge, and bridging veins, that may influence the clip application. Furthermore, the actual neck and dome conditions such as calcification, extreme wall thinning, and tightness between aneurysm dome and adherent vessels, are not indicated by the virtual intraoperative view. Strictly speaking, the success of complete clipping depends not only on the shape of arteries and aneurysms, but also on the thickness, stiffness, and other intrinsic factors of the walls.

The present simulation method can be used only for aneurysms with fundi directed laterally to the parent artery in the operative field and which are eligible for clipping with a straight clip. In the present study, false negative results were found for two aneurysms with wide necks, for which complete obliteration had been expected on the clipping simulation images. Therefore, there are many obstacles to apply a straight clip to the aneurysm neck. Nevertheless, preoperative prediction of aneurysm remnant after clipping can indicate more careful investigation of the blind corner using available modalities such as endoscopy and intraoperative angiography, and this can contribute to the complete obliteration of aneurysms. The future development of computer technology may improve the flexibility of clipping simulation.

\section{References}

1) Alexander TD, Macdonald RL, Weir B, Kowalczuk A: Intraoperative angiography in cerebral aneurysm surgery: a prospective study of 100 craniotomies. Neurosurgery 39: 10-18, 1996

2) Aoki S, Sasaki Y, Machida T, Ohkubo T, Minami M, Sasaki Y: Cerebral aneurysms: Detection and delineation using 3-D-CT angiography. AJNR Am J Neuroradiol 13: 1115-1120, 1992

3) Barrow DL, Boyer KL, Joseph GJ: Intraoperative angiography in the management of neurovascular disorders. Neurosurgery 30: 153-159, 1992

4) Carlotti CG Jr, Martelli N, Assirati JA Jr, Machado HR, Dos Santos AC, Colli BO: Subarachnoid hemorrhage after aneurysm surgery. Arq Neuropsiquiatr 54: 181-189, 1996

5) David CA, Vishteh AG, Spetzler RF, Lemole M, Lawton MT, Partovi S: Late angiographic follow-up review of surgically treated aneurysms. J Neurosurg 91: 396-401, 1999

6) Derdeyn CP, Moran CJ, Cross DT, Grubb RL Jr, Dacey RG Jr: Intraoperative digital subtraction angiography: a review of 112 consecutive examinations. AJNR Am J Neuroradiol 16: 307-318, 1995

7) Drake CG, Friedman AH, Peerless SJ: Failed aneurysm surgery. Reoperation in 115 cases. J Neurosurg 61: 848-856, 1984

8) Feuerberg I, Lindquist $C$, Lindquist $M$, Steiner L: Natural history of postoperative aneurysm rests. J Neurosurg 66: 30-34, 1987

9) Giannotta SL, Litofsky NS: Reoperative management of intracranial aneurysms. J Neurosurg 83: 387-393, 1995

10) Harrison M, Johnson BA, Gardner GM, Welling BG: Preliminary results on the management of unruptured intracranial aneurysms with magnetic resonance angiography and computed tomographic angiography. Neurosurgery 40: 947-957, 1997

11) Kamitani H, Masuzawa $H$, Kanazawa I, Kubo T: Bleeding risk in unruptured and residual cerebral aneurysms - angiographic annual growth rate in nineteen patients. Acta Neurochir (Wien) 141: 153-159, 1999

12) Kato Y, Sano H, Katada K, Ogura Y, Hayakawa M, Kanaoka N, Kanno T: Application of three-dimensional CT angiography (3D CTA) to cerebral aneurysms. Surg Neurol 52: 113-122, 1999

13) Kato $Y$, Sano H, Nagahisa S, Iwata K, Yoshida K, Yamamoto K, Kanno T: Endoscope-assisted microsurgery for cerebral aneurysms. Minim Invasive Neurosurg 43: 91-97, 2000

14) Lin T, Fox AJ, Drake CG: Regrowth of aneurysm sacs from residual neck following aneurysm clipping. J Neurosurg 70: 556-560, 1989

15) Martin NA, Bentson J, Vinuela F, Hieshima G, Reicher M, Black K, Dion J, Becker D: Intraoperative digital subtraction angiography and the surgical treatment of intracranial aneurysms and vascular malformations. J Neurosurg 73: 526-533, 1990

16) Nakase H, Kamada Y, Aoki H, Goda K, Morimoto T, Sakaki T: Clinical study on recurrent intracranial aneurysms. Cerebrovasc Dis 10: 255-260, 2000

17) Origitano TC, Schwartz K, Anderson D, Azar-Kia B, Reichman $\mathrm{OH}$ : Optimal clip application and intraoperative angiography for intracranial aneurysms. Surg Neurol 51: 117-128, 1999

18) Rauzzino MJ, Quinn CM, Fisher WS: Angiography after aneurysm surgery: indications for selective angiography. Surg Neurol 49: 32-41, 1998

19) Sakaki T, Takeshima T, Tominaga M, Hashimoto $H$, Kawaguchi S: Recurrence of ICA-PcoA aneurysms 
after neck clipping. J Neurosurg 80: 58-63, 1994

20) Sindou M, Acevedo JC, Turjman F: Aneurysmal remnants after microsurgical clipping: classification and results from a prospective angiographic study (in a consecutive series of 305 operated intracranial aneurysms). Acta Neurochir (Wien) 140: 1153-1159, 1998

21) Suzuki J, Kwak R, Katakura R: Review of incompletely occluded surgically treated cerebral aneurysms. Surg Neurol 13: 307-310, 1980

22) Tampieri D, Leblanc R, Oleszek J, Pokrupa R, Melancon D: Three-dimensional tomographic angiography of cerebral aneurysms. Neurosurgery 36: 749-755, 1995

23) Taniguchi M, Takimoto $H$, Yoshimine T, Shimada N, Miyao Y, Hirata M, Maruno M, Kato A, Kohmura E, Hayakawa T: Application of a rigid endoscope to the microsurgical management of 54 cerebral aneurysms: results in 48 patients. J Neurosurg 91: 231-237, 1999

24) Thornton J, Bashir Q, Aletich VA, Debrun GM, Ausman JI, Charbel FT: What percentage of surgically clipped intracranial aneurysms have residual necks? Neurosurgery 46: 1294-1300, 2000

25) Villablanca JP, Martin N, Jahan R, Gobin YP, Frazee J, Duckwiler G, Bentson J, Hardart M, Coiteiro D,
Sayre J, Vinula F: Volume-rendering helical computerized tomography angiography in the detection and characterization of intracranial aneurysms. J Neurosurg 93: 254-264, 2000

26) Vitaz TW, Gaskill-Shipley M, Tomsick T, Tew JM Jr: Utility, safety, and accuracy of intraoperative angiography in the surgical treatment of aneurysms and arteriovenous malformations. AJNR Am J Neuroradiol 20: 1457-1461, 1999

27) Yasargil MG: Anterior communicating artery aneurysms, in Yasargil MG (ed): Microneurosurgery II: Clinical Considerations, Surgery of the Intracranial Aneurysms and Results. New York, Thieme Stratton, 1984, pp 169-223

28) Zeman RK, Silverman PM, Vieco PT, Costello P: CT angiography. AJR Am J Roentgenol 165: 1079-1088, 1995

Address reprint requests to: K. Futami, M.D., Department of Neurosurgery, Toyama City Hospital, 2-1 Imaizumi-Hokubu-machi, Toyama 939-8511, Japan.

Commentary on this paper appears on the next page. 


\section{Commentary}

This is an excellent report by Futami and coworkers in Toyama and Kanazawa regarding the ability to predict clipping success (specifically 100\% clipping with no remnants of aneurysm neck) by a preoperative spiral CT and CT 3-D reconstruction. The reasoning is sound and the scientific design is appropriate. The authors simulated placement of a straight aneurysm clip with a line of sight technique on CT and predicted the operative findings (confirmed by direct observation, intraoperative angiography, or endoscopy).

There are several curious clinical points. All aneurysms were of the lateral wall type. Presumably, though not so stated, a study plan was made to use straight clips as the primary surgical strategy to facilitate comparison with CT imaging. The operating surgeon was not aware of the CT prediction. While this is scientifically valid, I wonder if the surgeon could have been aided by knowing the CT results in advance.

The results are excellent. There was high correlation between predicted and actual remnants. The authors do not report long-term consequences of such remnants, although this data is available elsewhere, and has been cited by the authors here. They are correct that remnant elimination must be achieved to maintain the superiority of surgical obliteration with intimal apposition over endosaccular filling.

The method reported has value in predicting potential remnants after aneurysm surgery. I suspect it is especially useful to select out those patients who should receive intraoperative angiography and/or endoscopy, which add time and risk to surgical procedures. The method is limited at present by the necessity for straight clipping, by the subset of aneurysms (lateral wall) suitable for study, and, as the authors state, by the inability to factor in other variables, such as neck thickness or calcification, in the decision analysis.

I congratulate the investigators on an excellent and well-conducted trial.

Christopher M. LofTus M.D., F.A.C.S. The Harry Wilkins Professor and Chairman Department of Neurological Surgery

The University of Oklahoma College of Medicine Oklahoma City, Oklahoma, U.S.A. 